\title{
Emotionsstörungen in der Rehabilitation
}

\section{Martin Lotze}

Emotion stammt vom lateinischen Wort „movere“ ab, das heißt „sich bewegen“. Wie diese Emotion die Disposition für eine Handlung bestimmt und wie sie sich in Motivation übersetzen lässt, davon berichtet dieser Artikel.

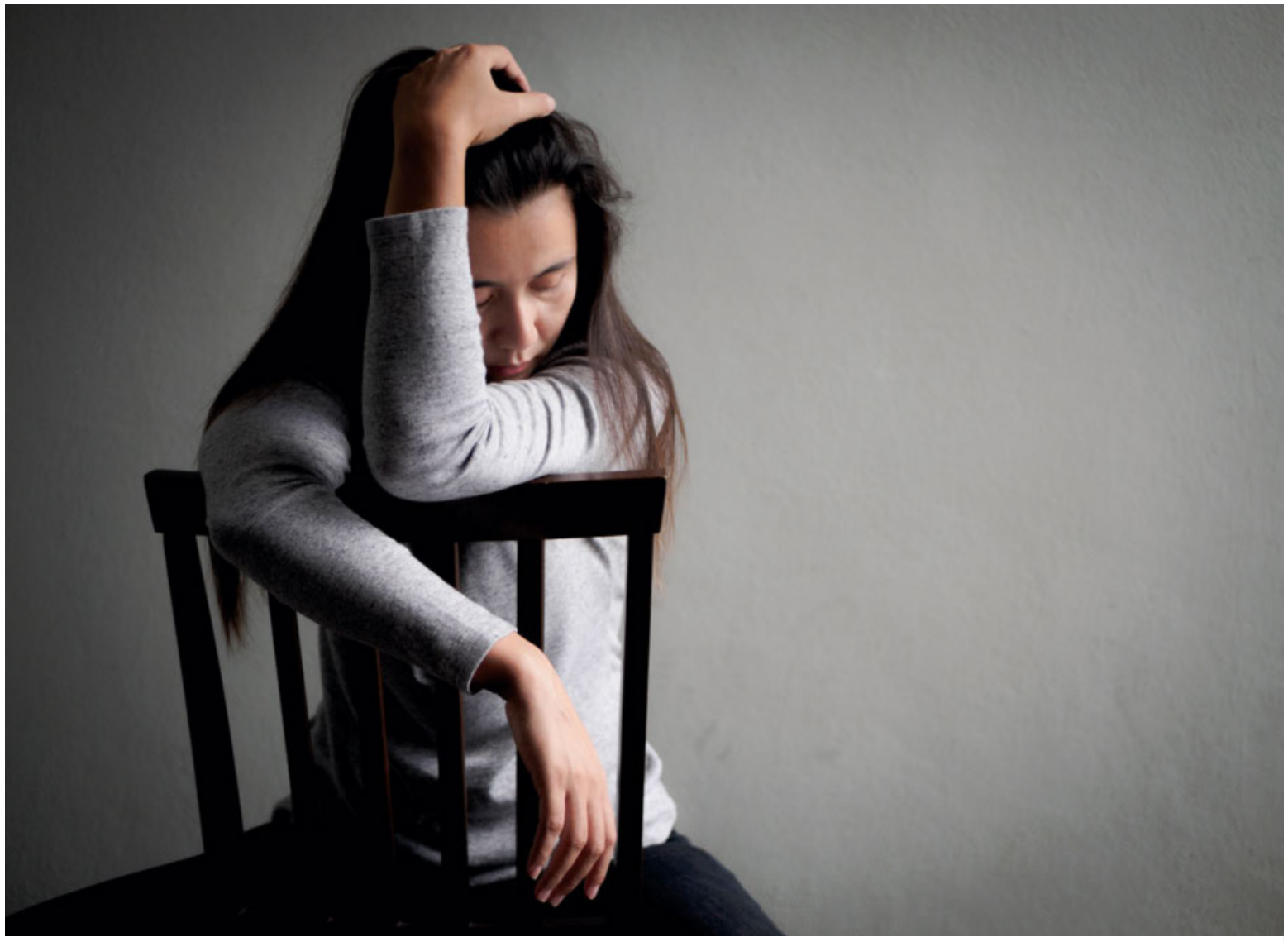

Störungen der Emotionalität treten bei einer Vielzahl von Erkrankungen des Zentralnervensystems auf. (Quelle: Siam/stock.adobe.com; nachgestellte Situation)

Der Körper ist mit seinen Ausdrucksbewegungen, den psychophysiologischen Veränderungen und seiner Haltung ein wesentlicher Aspekt der Emotionalität. Insgesamt kann man von drei Komponenten der Emotionalität ausgehen: der physiologischen Komponente (was kann man messen als Körperantwort? Anspannung, Schwitzen, Schreckreflex), der Wahrnehmungskomponente (wie nimmt der Proband die Emotion wahr?) sowie der Hand- lungskomponente (wie reagiert der Proband? Zum Beispiel mit Flucht oder Näherungsverhalten zu einem Objekt). Die Emotionalität bestimmt die Disposition für eine Handlung. Die innere Bewegung geht der Handlung also immer voraus - deshalb ist diese „Disposition“ so wichtig für die Neurorehabilitation. Eine solche Disposition kann man auch mit Motivation (z. B. für ein therapeutisches Training) übersetzen. 


\section{INFOBOX}

So werden Störungen der Emotionalität gemessen:

- Selbsteinschätzungsskalen oder Assessments (z. B. Self-Assessment Manikin; SAM zur Erfassung der Valenz und Erregung [4])

- Testungen (z. B. Einschätzen von emotionalem Ausdruck von Gesichtern)

- psychophysische Experimente (z. B. Hautleitfähigkeit, Schreckreflex, Herzrate)

- Experiment (z. B. klassische Konditionierung mit Erfassung der Wiederholungsrate für emotionales Lernen und Verlernen)

Obwohl sich Emotionalität in der Veränderung von Körperparametern abbildet, erfolgt ihre Steuerung im Gehirn. Deshalb treten Störungen der Emotionalität bei einer Vielzahl von Erkrankungen des Zentralnervensystems auf. Auch hier kann man von verschiedenen Ebenen der Schädigung von Emotionssteuerung ausgehen: die durch die Läsion selbst, durch eine Schädigung der Verbindung von Arealen oder eine Beeinflussung der Neurotransmitter im Gehirn. Emotionale Prozesse werden hierbei vor allem durch das Glukokortikoidsystem (z. B. Cortisol) und das sympathikoadrenerge System (z. B. Adrenalin) aktiviert (siehe auch [9]).

Will man eine grobe Unterteilung treffen, könnte man drei Syndromkomplexe für die Störungen der Emotionalität in der Neurorehabilitation identifizieren:

- fehlende Motivation, fehlender Antrieb und depressive Verstimmung

- Enthemmung und erhöhte Impulsivität

- spezifischere Störungen in der emotionalen Verarbeitung und Erkennung

\section{INFOBOX}

Insgesamt ist das Kardinalproblem von Patienten mit Störungen der Emotionalität eine soziale Isolation. Partner und Freunde erleben eine veränderte Emotionalität des Patienten und wenden sich danach oft ab. So ist die fehlende soziale Integration ein wichtiger Grund für eine schlechtere Prognose.

Direkte Störungen der Emotionsverarbeitung Sie entstehen durch Läsionen im emotionsverarbeitenden System („limbisches System“) und dessen Kontrolle (präfrontale Regulation, Exekutivkontrolle) und sind fast immer mit Läsionen der Verbindung zwischen diesen Regionen gekoppelt. Eher selten sind isolierte Ausfälle: Man könnte dies etwa bei der Zerstörung der linken anterioren Insel mit dem selektiven Ausfall des Schauders und der Freude an Musik nachweisen. Meist findet man keine umschriebene Symptomatik - die Patienten fallen oft erst später bei der fehlenden Wiedereingliederung mit sozialen Problemen auf. Zudem ist noch nicht geklärt, wie genau die In- teraktion einer physiologischen Veränderung und eines erlebten Gefühls vonstattengeht. Erleben wir Emotionen bewusst, weil unser Körper signalisiert, dass wir entsprechende Veränderungen aufzeigen? Dafür wären die viszeralen Afferenzen aber zu langsam, und wenn man sie im Tierversuch durchtrennt, kann das Tier dennoch emotional reagieren. Gibt es hier einen Monitor, der ständig die Veränderungen im Körper vergleicht? Es gibt Hinweise - gerade auch bei Patienten mit Schädigungen -, die so etwas in der Richtung nahelegen.

Somatic-Marker-Hypothese So ist die Somatic-Marker-Hypothese von Antonio Damasio [5] hier gut mit neurowissenschaftlichen Daten zusammenzubringen, bei denen eine enge Verbindung zwischen rascher Verarbeitung der Thalamus-Amygdala-Achse und zusätzlichen Eingängen aus den peripherphysiologischen Informationen (z. B. Propriozeption) im superioren Parietallappen postuliert wird.

Diese Hypothese basiert auf einer Untersuchung von Adolphs [2], bei der Patienten mit Läsionen im somatosensorischen Kortex, also im vorderen Parietallappen, Probleme hatten, Emotionen in Gesichtern zu erkennen. Es wurde vermutet, dass Patienten mit hochparietalen Läsionen wegen der mangelnden somatosensorischen Rückkopplung Probleme haben, Gefühlen „nachzuspüren“. Wird also bei Gesunden der visuell wahrgenommene Eindruck noch mal im somatosensorischen Programm „nachgespürt" bzw. gespiegelt?

Parietale Spiegelneurone Wahrscheinlich handelt es sich hierbei um parietale Spiegelneurone: Während Spiegelneurone im ventralen prämotorischen Kortex Bewegungen „spiegeln“ [13], könnten parietale Neurone somatosensorische Aspekte spiegeln. Eigene Körperzustände werden zudem ständig von der Insel gemonitort und tragen zur emotionalen Antwort bei. Wobei die Insel allein nicht notwendig ist, um Emotionen zu erleben [6]. Das Erleben eines Reizes addiert sich also aus den Komponenten Erwartung (Antizipation des Reizes), dem eigenen physiologischen Zustand und den verschiedenen sensorischen Informationen zu dem Objekt. Ein Schema für ein kombiniertes Modell des Somatic-Marker-Modells von Damasio mit der Darstellung der Monitorfunktion der Insel findet sich in - Abb. 1. Moduliert wird dieser Regelkreis zudem neuroendokrin, wobei die adrenerg-erregende Komponente besonders wesentlich ist.

\section{Bei welchen neurologischen Erkrankungen treten Störungen der Emotionalität auf?}

Zunächst sollte man bei Störungen der Emotionalität immer auch an eine Nebenwirkung einer bestehenden Medikation denken. Stimmungsschwankungen und An- 


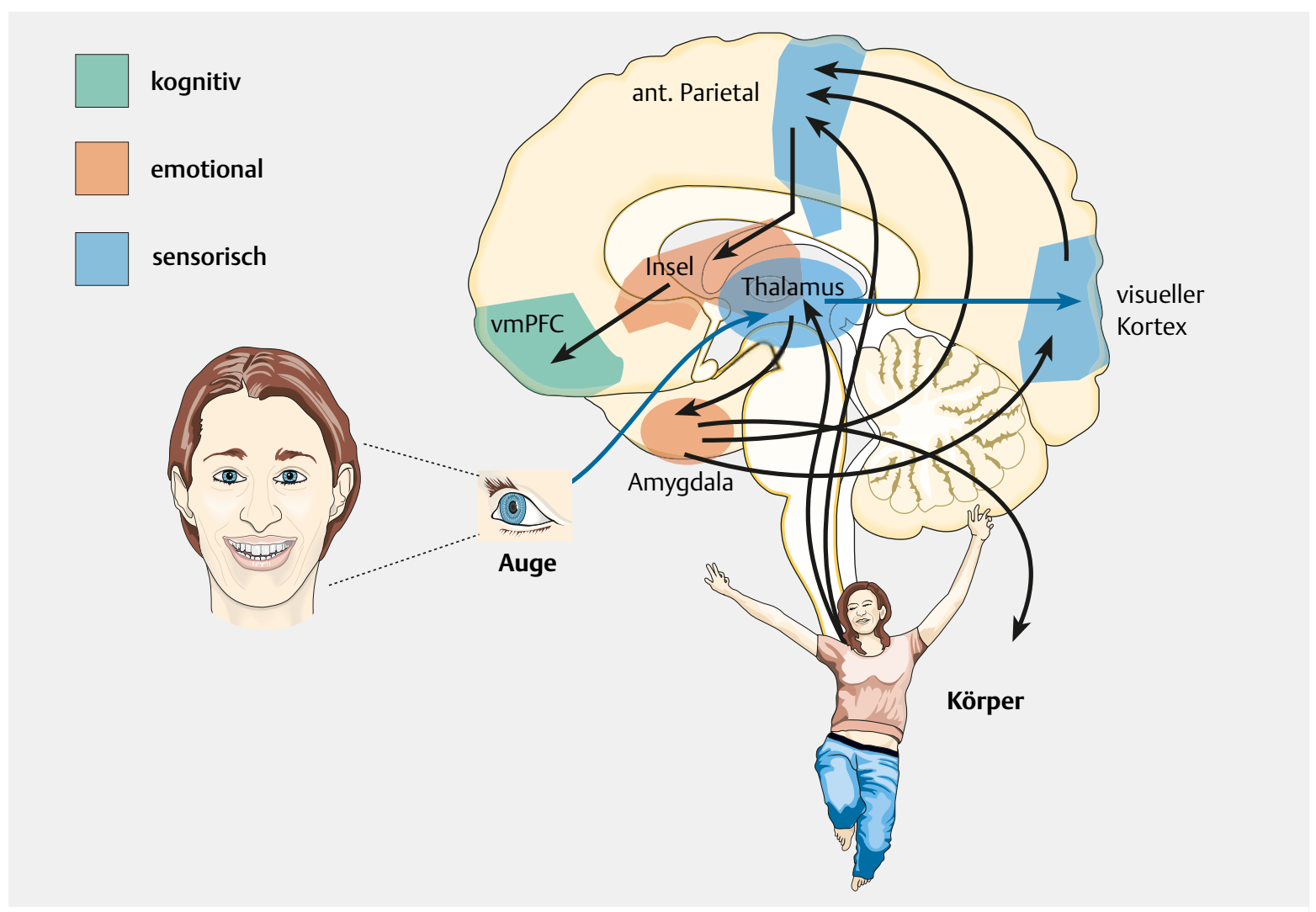

- Abb. 1 Erweitertes Modell, wie Körpersignale im Gehirn zum emotionalen Erleben beitragen könnten. Eine wahrgenommene mimische Emotion wird über den Sehnerv, den Thalamus und den visuellen Trakt in die Sehrinde weitergeleitet. Zudem gibt es einen raschen Verschaltungsweg über das Pulvinar thalami unter Modulation des visuellen Kortex direkt in die Amygdala. Über diesen Weg können schnelle Körperreaktionen (Rückzug oder Annäherung) initiiert werden. Informationen der Amygdala und aus dem Körper gelangen in den anterioren Parietalkortex, über den ein inneres Nachfühlen der Emotionen erfolgt. Zudem werden Informationen der Körperparameter in der Insel gemonitort und mit den kortikal parietalen Informationen integriert. Im ventromedialen präfrontalen Kortex (vmPFC) erfolgt erst dann eine kognitive Wertung der Emotion. (Quelle: M. Lotze; Umsetzung: Thieme Gruppe)

triebsstörungen sind häufige Begleiterscheinungen unterschiedlicher Medikamente.

Insgesamt zeigen große Läsionen im Frontalkortex einen Verlust der Exekutivkontrolle, Wesensänderungen und Antriebsstörungen, die bei beidseitiger Schädigung bis zum akinetischen Mutismus [1] gehen kann. Der Antriebsverlust ist hierbei bis zu einem kompletten Reaktionsverlust auf Umweltreize betroffen. Zudem treten bei orbitofrontalen und ventromedialen Schädigungen des Frontalhirns Wesensänderungen im Sinne einer Enthemmtheit auf. Diese umfangreichen Störungen, die vor allem bei Schädel-Hirn-Traumata oder beidseitigen Gefäßverschlüssen im anterioren Stromgebiet vorkommen, sind in dieser extremen Form selten.

Spezifische Schädigungen des emotionsverarbeitenden Systems treten vor allem bei den im Folgenden beschriebenen Erkrankungen auf.

\section{Schädel-Hirn-Trauma}

Wie im Kapitel zur Anatomie der Emotionsareale dargestellt wird, liegen diese in der Tiefe des Gehirns innerhalb und unterhalb des Frontal- und Temporallappens. Gerade Schädel-Hirn-Traumata (SHTs) können hier große Schäden durch traumatische Läsionen, bei denen weiche Gehirnmasse mit harter Schädelbasis oder frontal einwirkenden Objekten in Kontakt gerät, bewirken. Hier treten sowohl Zerstörungen des Frontalpols, des Temporalpols als auch Zerreißungen im Bereich der Verbindungen präfrontaler Exekutivkontrolle und limbischer Regionen auf. Insgesamt zeigen nach milden SHTs $45 \%$ der Patienten eine emotionale Beeinträchtigung, die zudem hohen prognostischen Wert hat [22]. Interessant ist zudem, dass eine Schädigung in der Fähigkeit, Gerüche wahrzunehmen (Dysosmie), die bei SHTs z. B. durch Scherungsverletzungen am Bulbus olfactorius oder in nachgeordneten Arealen beeinträchtigt sein kann, sehr häufig mit Störungen der Emotionalität einhergeht [15].

Aber nicht nur die Frontobasis ist entscheidend: Nach beidseitiger Schädigung des Temporalpols kommt es zum Klü- 
ver-Bucy-Syndrom mit der Neigung, jegliche Gegenstände in den Mund zu nehmen, sehr vertraut mit Fremden zu sein und zu exzessivem Sexual- und Essverhalten [20]. Dies kann jedoch auch durch andere Mechanismen, bei denen der Temporallappen geschädigt wird (epilepsiechirurgische Intervention, Herpesenzephalitis), hervorgerufen werden.

Insgesamt ist bei Patienten nach Schädel-Hirn-Trauma immer an eine Störung der Emotionalität zu denken, was wegen Verschlechterung der Prognose früh erkannt und therapeutisch berücksichtigt werden sollte.

\section{Störung der Emotionswahrnehmung und -produktion nach Schlaganfall}

Insbesondere rechtshemisphärische Schlaganfälle sind mit Störungen der Beurteilung emotionaler Reize wie etwa der Sprachmelodie assoziiert [18]. Auch in funktionell bildgebenden Studien sind diese oft mit Aktivität in rechtsorbitofrontalen Regionen assoziiert [23]. Bei einigen Patienten mit Schädigung im Bereich der inneren Kapsel und der Basalganglien tritt unkontrolliertes Weinen oder Lachen auf [19]. Hier scheinen Verbindungen, die diese Gefühlsausbrüche über das Mittelhirn kontrollieren, zerstört zu sein, sodass es zu einer Enthemmung kommt.

Drei Monate nach einem Schlaganfall entwickelt jeder dritte Patient eine depressive Störung (Post-Stroke Depression; PSD). Damit assoziiert sind häufig Einschränkungen im Alltag sowie Einschränkungen des sozialen und kognitiven Funktionsniveaus. PSD ist durch eine über mindestens zwei Wochen anhaltende depressive Verstimmung oder den Verlust an Interesse oder Freude gekennzeichnet. Zudem treten weitere Symptome auf, wie z. B. Schlafstörungen, veränderter Appetit, Schuldgefühle oder Antriebslosigkeit. Patienten mit PSD haben später höhere Einschränkungen im täglichen Leben, zeigen verminderte Mobilität und Teilhabe am Alltag [21] und weisen eine deutlich erhöhte Mortalität 1-5 Jahre nach Schlaganfall auf.

Befunde zum Effekt des Läsionsortes sind nach wie vor widersprüchlich. Es gibt insgesamt Hinweise, dass präfrontale Läsionen eher mit einer depressiven Symptomatik assoziiert sind (neuste Übersicht in [16]). Schon länger wird diskutiert, ob generelle Effekte wie vaskuläre Minderperfusion alleine bereits zu depressiven Störungen führen kann [3]. Schädigungen des Neurotransmittersystems durch den Schlaganfall, z. B. des serotonergen Systems, sind ebenso nachgewiesen worden und tragen sicher zur Symptomatik bei. So zeigen kortikale und striäre Läsionen häufig eine Minderung der Serotoninkonzentration im Urin [8].

Obwohl Antidepressiva zur Behandlung und Prävention von PSD günstige Effekte zeigen, wird die pharmakologische Behandlung von PSD zwar empfohlen, aber auch kritisch diskutiert. Die Nebenwirkungen, etwa bei Selektiven Serotoninwiederaufnahmehemmern, sind bei multimorbi- den Schlaganfallpatienten besonders wesentlich, weil sie vor allem das Kreislaufsystem betreffen: ein erhöhtes Risiko für hämorrhagische Komplikationen, für einen Myokardinfarkt, für einen erneuten Schlaganfall sowie ein erhöhtes Sturzrisiko bei Senioren.

Achtsamkeitstherapie ist auch bei der Behandlung der PSD auf dem Vormarsch: Erste Untersuchungen mit Schlaganfallpatienten konnten positive Effekte auf erlebte Angst, Depression, Fatigue sowie auf Blutdruck und Lebensqualität nachweisen [11]. Auf die bisherigen Cochrane-basierten Evidenzen geht der Artikel zu psychischen Begleiterkrankungen nach Schlaganfall detailliert ein [7].

\section{Multiple Sklerose}

Noch schwieriger wird es bei Störungen, die sowohl mit Entzündungsherden als auch mit Atrophie des Gehirns einhergehen, wie die Multiple Sklerose (MS). Da diese disseminiert auftreten, kommt es zu unsystematischen Beeinträchtigungen auch der Emotionalität. Hierbei kann es zu Emotionserkennungsstörungen kommen, die eine adäquate emotionale Reaktion in der sozialen Interaktion beeinträchtigen können [10].

Insbesondere die Fatigue ist ein bei der MS sehr häufig vorkommendes Syndrom, das neuropsychologisch oft schwer von einer Depression abgrenzbar ist. Die Patienten klagen über ein überwältigendes Gefühl von Erschöpfung und Müdigkeit, mangelnden Antrieb, bei nächtlicher Schlafstörung und Stimmungsreduktion. Ursächlich wird eine Störung der Hypothalamus-Hypophysen-Adrenalin-Achse sowie verminderte Serotoninverfügbarkeit bei diesen Patienten vermutet. Man kann sich aber auch vorstellen, dass diese Mechanismen sekundär durch Bewegungsverlust induziert werden. Zudem sind die Symptome sehr oft pharmakologisch verursacht, wobei dies besonders oft bei Antikonvulsiva, Antidepressiva und Schmerzmedikamenten vorkommt.

Schließt man periphere Erkrankungen (Muskelerkrankungen; Polyneuropathie) aus, kann Fatigue auch über die mangelnde Initiative zur Bewegung oder zum Durchhalten von Bewegungen gemessen werden. Oft besteht bei Parkinson-Erkrankung und MS eine Dissoziation zwischen der Fatigue und den objektivierbaren Beeinträchtigungen in der kognitiven Leistungsfähigkeit. Ein multidimensionaler Fragebogen für kognitive und motorische Einschränkungen ist die Fatigue Scale for Motor and Cognitive Functions (FSMC; [17]). Andere Fragebögen erscheinen hinsichtlich spezifischer Erkrankungen geeignet (Profile of Mood States Fatigue Subscale; [14]).

Therapeutisch sind pharmakologische Strategien wenig erfolgreich. Vielversprechender sind individuell angepasste Verfahren: Bei MS ist das die Kältetherapie (Abkühlen des gesamten Körpers), Entspannungsverfahren (Autogenes Training, Muskelrelaxation), aber auch kognitive Ver- 
fahren wie Bewegungsvorstellung und Achtsamkeitstraining.

\section{Affektive Störungen bei Morbus Parkinson}

Während Perzeptionsstörungen von emotionaler Intensität bei Mimik in einer kleinen Gruppe von Patienten mit der Dopaminverfügbarkeit einherging [12], fand sich hinsichtlich der Affektstörung eine Dissoziation zwischen der motorischen Produktion und der Intensität der Depression. Aus diesem Grund ist hier der Mangel an Dopamin nicht im Vordergrund - durch Dopaminsubstitution ändert sich die Depression häufig nicht so, dass ein zusätzliches Serotonindefizit vermutet wird [1]. Bei manchen Patienten (ca. 7\%) tritt unter der Dopaminsubstitutionstherapie eine pathologische Spielsucht auf. Hier werden zusätzliche Dopaminrezeptoren (D3) überstimuliert. Nach Absetzen oder Umstellung der Medikation ist diese Nebenwirkung in der Regel reversibel.

\section{Emotionsstörungen nach neurochirurgischen Interventionen}

Vor allem die Epilepsiechirurgie mit Teilresektion medialer Temporallappenanteile kann hier Probleme bereiten. Patienten mit etwa epilepsiechirurgischer Resektion der Amygdala haben Einschränkungen bei der Gesichtsverarbeitung, vor allem in Bezug auf ängstliche und ärgerliche Gesichtsausdrücke [2]. Zudem können Störungen des emotionalen Lernens auftreten. Neurochirurgische Interventionen nach Resektion von Tumoren sollten hinsichtlich Schädigung des limbischen Systems, kortikaler Kontrolle von Emotionen und deren Verbindungen auf das Risiko einer Emotionsstörung hin kritisch diagnostisch getestet werden. Eine frühzeitige Erfassung der Störung kann sekundäre soziale Probleme deutlich begrenzen.

\section{Schlussbemerkung}

Insgesamt sind Emotionsstörungen in der Neurorehabilitation zu wenig beachtet, obwohl sie häufig auftreten. Da neurorehabilitative Therapie und Prognose bei zusätzlich bestehenden emotionalen Störungen ungünstig verlaufen, ist ein professionellerer Umgang in der Erkennung und der Therapie dieser Störungen angezeigt. Es sollte frühzeitig an diese Störungen und deren Vorkommen gedacht, eine Beeinträchtigung getestet und es sollten rechtzeitig Maß- nahmen eingeleitet werden, die sozialen Probleme, die durch die Störung der Emotionalität hervorgerufen werden, zu vermeiden. Hierbei reichen pharmakologische Interventionen meistens nicht aus, sondern es sollte über verschiedene Ebenen therapiert werden. Insbesondere verhaltenstherapeutische Interventionen, Feedbackverfahren sowie kognitive und entspannende Verfahren zeigen vielversprechende Ansätze und sollten in Therapiestudien weiter hinsichtlich Indikation und Wirksamkeit geprüft werden. Zudem sind regelnde Verfahren bzw. auch Verfahren zur Sicherung der Betreuenden (besonders bei erhöhter Impulsivität der Patienten) angebracht.

\section{Autorinnen/Autoren}

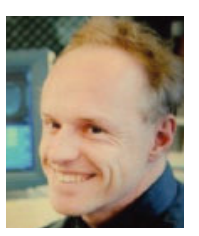

Martin Lotze

Martin Lotze ist Professor für Funktionelle Bildgebung und Neurologie an der Universität Greifswald. Er forscht unter anderem über neuronale Effekte bei Training, neuronale Plastizität und chronische Schmerzen.

\section{Korrespondenzadresse}

Prof. Dr. Martin Lotze

Ernst-Moritz-Arndt-Universität Greifswald

Walter-Rathenau-Str. 46

17475 Greifswald

E-Mail: martin.lotze@uni-greifswald.de

\section{Literatur}

Die komplette Literaturliste finden Sie unter www.thieme-connect.de/products

Bibliografie

DOI https://doi.org/10.1055/a-0596-6654 neuroreha 2018; 10: 61-65

(c) Georg Thieme Verlag KG Stuttgart - New York ISSN 1611-6496 